\title{
Quantum manipulations of small Josephson junctions and the
} measurement process performed with a single-electron transistor

\author{
Alexander Shnirman ${ }^{\mathrm{a}}$, Yuriy Makhlin ${ }^{\mathrm{b}, \mathrm{c}}$ and Gerd Schön ${ }^{\mathrm{b}}$ \\ aDept. of Physics, University of Illinois at Urbana-Champaign, Urbana, IL 61801-3080, \\ U.S.A. \\ bInstitut für Theoretische Festkörperphysik, Universität Karlsruhe, D-76128 Karlsruhe, \\ Germany \\ ${ }^{c}$ Landau Institute for Theoretical Physics, 117940 Moscow, Russia
}

Nano-scale electronic devices provide physical realization of the elements required for quantum computation [1-3]. Small Josephson junctions, where Coulomb blockade effects allow the control of individual Cooper-pair charges, constitute quantum bits (qubits), with logical states differing by the charge on one island. Single- and two-bit operations can be performed by applied gate voltages. The phase coherence time is sufficiently long to allow a series of these steps. In addition to the manipulation of qubits the resulting quantum state has to be read out. This can be accomplished by coupling a single-electron transistor to the qubit [4]. We describe this quantum measurement process by considering the time-evolution of the density matrix of the coupled system. The transistor destroys the phase coherence of the qubit only when a transport voltage is turned on. The process is characterized by three time scales: the dephasing time, the 'measurement time' when the signal resolves the different quantum states, and the mixing time after which the measurement process itself destroys the information about the initial state.

\section{Introduction}

The investigation of nano-scale electronic devices, such as low-capacitance tunnel junctions or quantum dot systems, has always been motivated by the perspective of future applications. By now several have been demonstrated, e.g. the use of SETs as ultrasensitive electro-meters and single-electron pumps. From the beginning it also appeared attractive to use these systems for digital operations needed in classical computation [5]. Obviously single-electron devices would constitute the ultimate electronic memory. Unfortunately, their extreme sensitivity makes them also very susceptible to fluctuations, either due to the external circuit or microscopic sources such as electron hopping in the substrate. Due to these problems - and the continuing progress of conventional techniques - the future of SET devices in classical digital applications remains uncertain.

The situation is different when we turn to elements for quantum computers. They could perform certain calculations which no classical computer could do in acceptable times by exploiting the quantum mechanical coherent evolution of superpositions of states [6]. Here conventional systems provide no alternative. In this context, ions in a trap, manipulated 


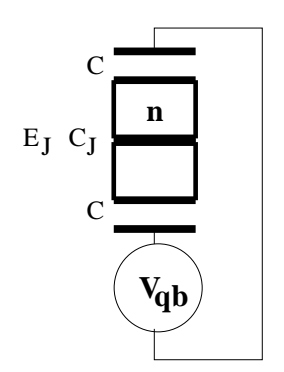

a)

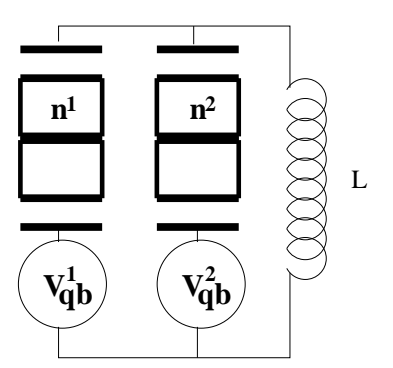

b)

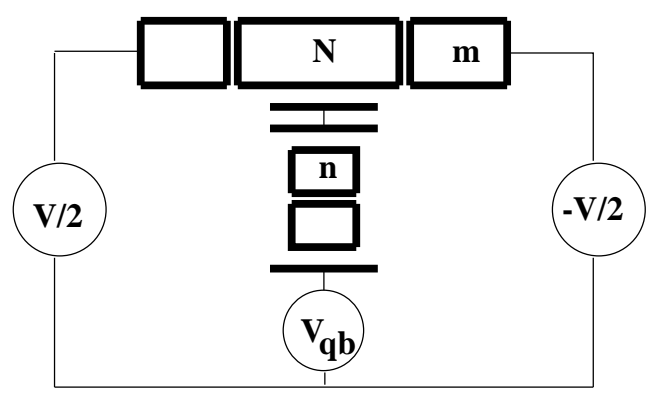

Figure 1. The proposed 1-bit and 2-bit Josephson systems.

Figure 2. The circuit consisting of a qubit plus a SET used as a measuring device.

by laser irradiation are the best studied system. However, alternatives need to be explored, in particular those which are more easily embedded in an electronic circuit. From this point of view nano-electronic devices appear particularly promising.

The simplest choice, normal-metal single-electron devices are ruled out, since - due to the large number of electron states involved - different, sequential tunneling processes are incoherent. Ultra-small quantum dots with discrete levels and spin degrees of freedom are candidates [3], but the strong coupling to the environment renders the phase coherence time short. More attractive are systems of Josephson contacts, where the coherence of the superconducting state can be exploited. Quantum extension of elements based on a single flux logic have been suggested, however, an essential process, the coherent oscillation of the flux between degenerate states, has not yet been observed. We suggest the use of low-capacitance Josephson junctions, where Cooper-pair charges tunnel coherently and can be controlled by applied gate voltages [1].

In addition to controlled manipulations the quantum computation requires a quantum measurement to read out the final state. The requirements for both steps appear to contradict each other. During manipulations the dephasing should be minimized, whereas a measurement should dephase the state of the qubit as fast as possible. The option to couple the measuring device to the qubit only when needed is hard to achieve in nanoscale systems. The alternative, which we suggest [4], is to keep the measuring device permanently coupled to the qubit in a state of equilibrium during the quantum operations. The measurement is performed by driving the measuring device out of equilibrium, which dephases the quantum state of the qubit. Similar nonequilibrium dephasing processes [7] have recently been demonstrated experimentally [8].

\section{Josephson junction qubits}

First we discuss the properties and quantum manipulations of Josephson qubits shown in Fig. 1. Each qubit consists of two superconducting islands coupled by a Josephson junction. The coupling to the external circuit is purely capacitive and does not involve dissipative currents. The qubit's state is characterized by $n$, the number of extra Cooper pairs on the upper island. The scale of the energy splitting between consecutive charge states is the charging energy $E_{\mathrm{qb}}$; the precise value can be tuned by an applied gate 
voltage $V_{\mathrm{qb}}$. We concentrate here on the regime where the charging energies of two states, $|\downarrow\rangle \equiv|n\rangle$ and $|\uparrow\rangle \equiv|n+1\rangle$, are close. Choosing the Josephson coupling sufficiently weak, $E_{\mathrm{J}} \ll E_{\mathrm{qb}}$, we ensure that the coherent Cooper-pair tunneling only mixes these two states. The effective Hamiltonian thus reduces to $H=\frac{1}{2} E_{\mathrm{J}}\left(\cot \eta \sigma_{z}+\sigma_{x}\right)$. The mixing angle $\eta$ depends on the ratio of energy scales $E_{\mathrm{J}} / E_{\mathrm{qb}}$ and the applied gate voltage $V_{\mathrm{qb}}$. Here it is sufficient to note that the latter allows the tuning of $H$.

The system is thus equivalent to a spin in a magnetic field with constant $x$-component, while the $z$-component can be varied by $V_{\mathrm{qb}}$. Hence, the standard techniques of spin manipulations by time-dependent fields $(\pi / 2$-pulses, ...) are available for manipulations of the qubit [1]. By varying the gate voltage we can put the system in a regime where the mixing is strong, which is of advantage when performing quantum manipulations. On the other hand, between manipulations and we keep it at the "idle point", $\eta_{\text {idle }}\left(\sin \eta_{\text {idle }} \ll 1\right)$, far from degeneracy. The eigenstates of the qubit at the operation point constitute its logical basis: $|0\rangle=\cos \frac{\eta_{\text {idle }}}{2}|\downarrow\rangle+\sin \frac{\eta_{\text {idle }}}{2}|\uparrow\rangle$ and $|1\rangle=-\sin \frac{\eta_{\text {idle }}}{2}|\downarrow\rangle+\cos \frac{\eta_{\text {idle }}}{2}|\uparrow\rangle$. The energy splitting between the logical states is then $\Delta E \equiv E_{\mathrm{J}} / \sin \eta_{\text {idle }} \gg E_{\mathrm{J}}$.

Since the splitting $\Delta E$ is non-zero, the relative phase of two components of the state evolves between manipulations (the spin precesses). The state is preserved, however, in the interaction representation (in terms of spins, in the rotating frame). In this representation, if the voltage is changed at $t_{0}$ for some time $\tau$ to the value $V$ defining a new $\eta$, the quantum state of the qubit evolves according to the unitary transformation $\mathcal{U}\left(t_{0}, \tau, \eta\right)=$ $\exp \left(i H\left(\eta_{\text {idle }}\right)\left(t_{0}+\tau\right)\right) \cdot \exp (-i H(\eta) \tau) \cdot \exp \left(-i H\left(\eta_{\text {idle }}\right) t_{0}\right)$. With proper choice of $t_{0}, \tau$, and $V$ any 1 -bit operation can be realized. E.g., $\mathcal{U}\left(t_{0}, \tau=\pi \hbar \cos \eta_{\text {idle }} / E_{\mathrm{J}}, \eta=\eta_{\text {idle }} \pm \pi / 2\right)$ is a NOT operation, exchanging $|0\rangle$ and $|1\rangle$, while $\mathcal{U}\left(t_{0}, \tau=2 \pi N \hbar \sin \eta / E_{\mathrm{J}}, \eta\right)$ is a shift of their relative phase by $\phi=2 \pi N \sin \eta / \sin \eta_{\text {idle }}$ for any integer $N$.

To perform 2-bit operations, we couple the qubits, as shown in Fig. 1b, by an oscillatory mode of an inductance $L$ and the total capacitance of the qubits. The oscillator frequency should be much larger than $\Delta E$. Then, the oscillator stays in the ground state, while its zero-point fluctuations induce a coupling between the qubits of the form $E_{L} \sigma_{y}^{1} \sigma_{y}^{2}$. The energy scale $E_{L} \propto E_{\mathrm{J}}^{2} L / \Phi_{0}^{2}$, with $\Phi_{0} \equiv h / 2 e$ being the flux quantum, should be small compared to $\Delta E$. When, by varying the gate voltages, the states $\left|\downarrow^{1}, \uparrow^{2}\right\rangle$ and $\left|\uparrow^{1}, \downarrow^{2}\right\rangle$ are tuned close to degeneracy, this interaction produces coherent "flip-flop" transitions. One can compensate for the effect of unwanted phase shift using single-bit operations. Away from degeneracy, the inductor produces only a weak perturbation for each qubit.

The electromagnetic circuit induces fluctuations of the bias voltage $V_{\mathrm{qb}}$, which couple to the charge of the qubit $\sigma_{z}$. As a result the diagonal elements of the density matrix, in the basis of the qubit's eigenstates, relax to their equilibrium values with a rate $\Gamma_{\text {incoh }}$, while the off-diagonal elements decay with the dephasing rate $\Gamma_{\phi}$. In the low-frequency regime the system consisting of qubit and environment is described by the spin-boson model at finite bias [9], and we can take over the established results. When the qubit is kept at the idle point the two rates are $\Gamma_{\text {incoh }}=\frac{\pi}{\tilde{g}} \frac{\Delta E}{\hbar} \operatorname{coth}\left(\frac{\Delta E}{2 k_{B} T}\right) \sin ^{2} \eta_{\text {idle }}$ and $\Gamma_{\phi}=\frac{1}{2} \Gamma_{\text {incoh }}+$ $\frac{2 \pi}{\tilde{g}} \frac{k_{B} T}{\hbar} \cos ^{2} \eta_{\text {idle. }}$. The parameter $\tilde{g} \approx\left(C_{\mathrm{J}} / C\right)^{2} h /\left(4 e^{2} R\right)$ characterizes the coupling to the electromagnetic environment, model here by a resistor $R$. The rate $\Gamma_{\text {incoh }}$ corresponds to real transitions between the two eigenstates of the qubit, while the second term in the expression for $\Gamma_{\phi}(\propto T)$ originates from the fluctuations of the energy splitting between the 
eigenstates. The last process may be called a "pure" dephasing since it occurs even if there is no tunneling between the islands of the qubit $\left(E_{\mathrm{J}}=0\right)$, i. e. in the absence of transitions. The decay rates are small if the resistance of the circuit is low compared to the quantum resistance, $h / e^{2} \approx 26 \mathrm{k} \Omega$. Furthermore, a low gate capacitance $C$ reduces the coupling of the qubit to the environment. With suitable parameters $\left(R \leq 100 \Omega, C / C_{\mathrm{J}} \leq 0.1\right)$ at low temperatures the number of operations which can be performed before the environment destroys the coherence may be as large as $10^{3}-10^{4}$.

\section{The quantum measurement process}

The most elementary system proposed for the quantum measurements is shown in Fig. 2. It is a SET, with a qubit inserted into the control gate circuit. At the stage of the quantum manipulations the SET is kept deeply in the off-state $(N=0)$, no dissipative currents flow in the system, and dephasing effects due to the transistor are minimized. When one drives the SET out of equilibrium, the resulting normal current depends on the state of the qubit, since different charge configuration induce different voltages on the middle island of the SET. The picture gets complicated by various noise factors (shot noise) and by the measurement induced transitions between the states of the qubit. The first set the lower time limit after which we can extract the information from the experimental data, while the last destroy the information of the quantum state to be measured.

The total system is characterized by three charging energies: the typical energy splitting between consecutive charge states $(N)$ of the SET, $E_{\text {set }}$, the charging energy scale of the qubit, $E_{\mathrm{qb}}$, and the Coulomb repulsion between charges on the qubit and the middle island of the SET, $E_{\text {int }}$. To drive the system out of equilibrium one applies a transport voltage $V$, so that tunneling to another charge state, say $N=1$, becomes possible. We choose $E_{\text {set }}$ to be the largest energy scale, $E_{\text {set }} \gg \Delta E \gg E_{\mathrm{J}}, E_{\text {int }}$, and the transport voltage large enough to overcome the Coulomb energy gap between the states $N=0$ and $N=1$ by an amount of order $E_{\text {set }}$, but small enough to insure that only these two states are involved in the transport. The actual value of the Coulomb gap differs by $E_{\text {int }}$ for different charge states of the qubit. As a result, an electron finds it easier to tunnel to the middle island of the SET when there are less Cooper pairs on the upper island of the qubit. Thus, the total transport rates $\Gamma_{0}$ and $\Gamma_{1}$, corresponding to the qubit's states $|0\rangle$ and $|1\rangle$, differ by $2 \pi \alpha E_{\text {int }}$, where $\alpha \equiv \hbar /\left(4 \pi^{2} e^{2} R_{\mathrm{T}}\right)$ characterizes the tunneling resistance of the normal tunnel junctions. Conversely, when the SET is in the state $N=1$, the energy splitting between the states $|0\rangle$ and $|1\rangle$ is shifted by $E_{\text {int }}$ and, therefore, an additional relative phase is acquired. Since the tunneling events in the SET are random, they are a source of dephasing. Moreover, when $N=1$, the states $|0\rangle$ and $|1\rangle$ are no longer the exact eigenstates. This gives rise to the measurement induced transitions.

To describe the dissipative current in the SET we introduce the variable $m$ which counts the number of electrons which has arrived in the right lead. Thus the total system is described by a reduced density matrix $\hat{\rho}_{i, N, m ; i^{\prime}, N^{\prime}, m^{\prime}}(t)$, where $i$ and $i^{\prime}$ stand for the quantum states of the qubit, $|0\rangle$ or $|1\rangle$, the variables $N$ and $m$ have been introduced above, and all other degrees of freedom are traced out. The off-diagonal elements in $N$ and $m$ may be eliminated from a closed set of equations [10]. Therefore, we need

to consider only the elements $\hat{\rho}_{i, i^{\prime}}^{N, m} \equiv \hat{\rho}_{i, N, m ; i^{\prime}, N, m}$. We assume in the following that at 
time $t=0$, as a result of previous quantum manipulations, the qubit is prepared in the superposition state $a|0\rangle+b|1\rangle$, and we switch on a transport voltage to the SET.

To proceed we further reduce the density matrix in two ways to obtain dual descriptions of the measurement. The first procedure is to trace over $N$ and $m$, which yields a reduced density matrix of the qubit $\hat{\rho}_{i, j} \equiv \sum_{N, m} \hat{\rho}_{i, j}^{N, m}$. Starting in a superposition of states $\left(\hat{\rho}_{0,0}=\right.$ $\left.|a|^{2}, \hat{\rho}_{1,1}=|b|^{2}, \hat{\rho}_{0,1}=a b^{*}\right)$ the questions are how fast the off-diagonal elements of $\hat{\rho}_{i, j}$ vanish due to dephasing, and how fast the diagonal elements change their original values (for instance due to transitions induced by the measurement). This description is enough when one is interested in the quantum properties of the qubit only and the measuring device is used as a source of dephasing [7,8]. It does not tell us much, however, about the quantity measured in an experiment, namely the current flowing trough the SET.

The second procedure is to evaluate the probability distribution of the number of electrons $m$ which have tunneled trough the SET during time $t: P(m, t) \equiv \sum_{N, i} \hat{\rho}_{i, i}^{N, m}(t)$. At $t=0$ no electrons have tunneled, so $P(m, 0)=\delta_{m, 0}$. Then this peak starts to shift towards positive $m$, and, at the same time, it widens due to shot noise. Since two states of the qubit correspond to different conductivities, one may hope that after some time the peak splits into two. If after sufficient separation of the two peaks their weights are still close to $|a|^{2}$ and $|b|^{2}$, a good quantum measurement has been performed. Unfortunately, there exist processes which destroy this idealized picture. After a long time the two peaks transform into a broad plateau, since transitions between the qubit's states are induced by the measurement. One should find an optimum time for the measurement, so that, on one hand, the two peaks are separate and, on the other hand, the induced transitions have not yet happened. To this end we derive a master equation for $\hat{\rho}_{i, j}^{N, m}$, using the real time diagrammatic technique [10,4], and we analyze it both analytically and numerically.

First, we assume that the Josephson coupling is switched off during the measurement. Then the master equation splits into three independent groups of equations: two for the diagonal matrix elements $\hat{\rho}_{0,0}^{N, m}$ and $\hat{\rho}_{1,1}^{N, m}$ and the third for the off-diagonal elements. The first two groups describe propagation of two independent peaks with velocities $\Gamma_{0}$ and $\Gamma_{1}$. The weights of the peaks are $|a|^{2}$ and $|b|^{2}$ and their widths at time $t$ are given by $\sqrt{\Gamma_{0} t}$ and $\sqrt{\Gamma_{1} t}$ (shot noise). Thus one arrives at the criterion for the peaks' separation: $\left|\Gamma_{0}-\Gamma_{1}\right| t \geq \sqrt{\Gamma_{0} t}+\sqrt{\Gamma_{1} t}$, which yields the time of measurement: $t_{\mathrm{ms}}=\left|\sqrt{\Gamma_{0}}-\sqrt{\Gamma_{1}}\right|^{-2}$.

The third group of equations describes the decay of the off-diagonal matrix elements $\hat{\rho}_{0,1}^{N, m}$. We find the dephasing time $\tau_{\phi}$ to be parametrically different from the measurement timet $t_{\mathrm{ms}}$. In the range of validity of our approach $t_{\mathrm{ms}}$ exceeds $\tau_{\phi}$, consistent with the fact that a quantum measurement implies a complete dephasing of a quantum state. The faster dephasing indicates that during the measurement some additional uncontrolled parts of the environment "observe" the quantum state of the qubit. For example, information may be lost into the unspecified microscopic states of the SET.

The residual Josephson coupling ignored so far generates mixing transitions between the states of the qubit. We estimate the mixing time for the concrete physical situation discussed above $t_{\text {mix }}^{-1} \approx 2 \pi \alpha E_{\text {int }}^{2} E_{\mathrm{J}}^{2} E_{\text {set }} /(\Delta E)^{4}$, while the measurement time is given approximately by $t_{\mathrm{ms}}^{-1} \approx 2 \pi \alpha E_{\mathrm{int}}^{2} / E_{\mathrm{set}}$. The results of numerical simulations of the master equation in the regime $t_{m s} \ll t_{m i x}$, showing the peak separation, are presented in Fig. 3 .

Here we have described that the current through a SET measures the quantum state of the qubit, in the sense that for a superposition of two eigenstates it yields one or 


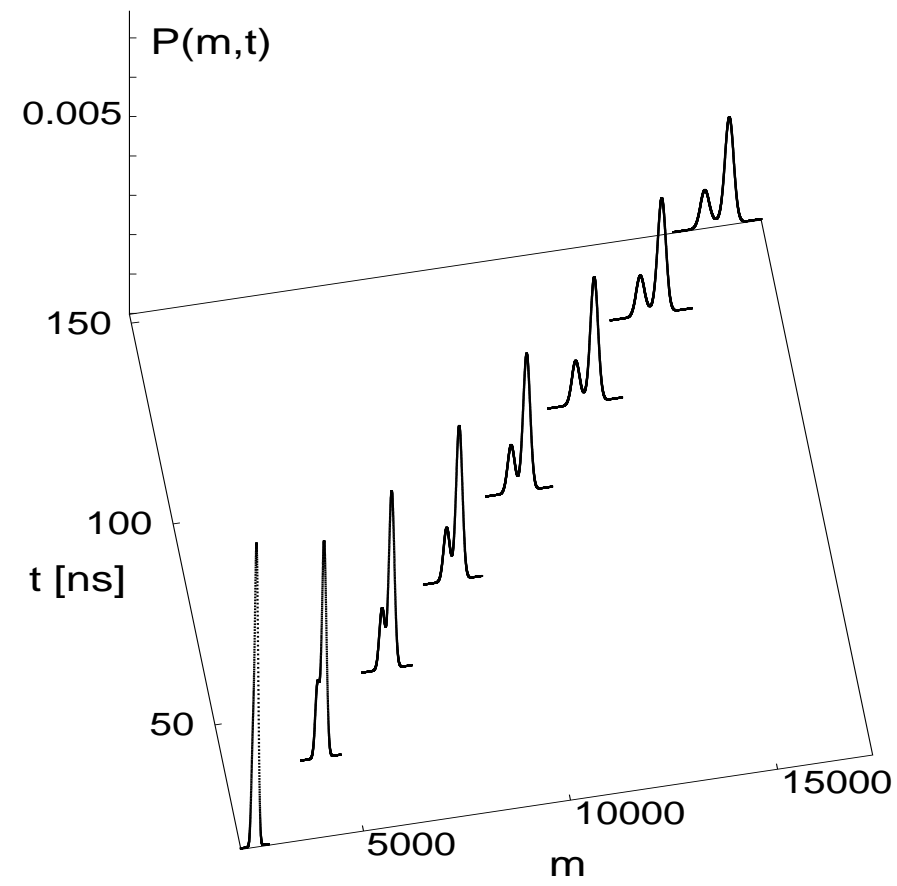

Figure 3. $P(m, t)$, the probability that $m$ electrons have tunneled during time $t$ (measured in nano-seconds). The initial amplitudes of the qubit's states are $a=\sqrt{0.75}, b=\sqrt{0.25}$.

the other result with the appropriate probabilities. This should be distinguished from another question, namely whether it is possible to demonstrate that an eigenstate of a qubit can actually be a superposition of two different charge states. The latter had been addressed successfully in the experiments of Refs. [11] where, in a setup similar to Fig. 2, a single-Cooper-pair box was coupled to a SET.

\section{REFERENCES}

1. A. Shnirman, G. Schön, and Z. Hermon, Phys. Rev. Lett. 79, 2371 (1997).

2. J.E. Mooij, private comm.; D.V. Averin, Solid State Commun. 105, 659 (1998).

3. D. Loss and D.P. DiVincenzo, Phys. Rev. A 57, 120 (1998); G. Burkard et al., condmat/9808026.

4. A. Shnirman and G. Schön, Phys. Rev. B 57, 15400 (1998).

5. A.N. Korotkov, R.H. Chen, and K.K. Likharev, J. Appl. Phys. 78, 2520 (1995).

6. A. Barenco, Contemp. Phys. 37, 375 (1996); D.P. DiVincenzo, in Mesoscopic Electron Transport, NATO ASI Series E 345, eds. L.L. Sohn et al., Kluwer, 1997.

7. Y. Levinson, Europhys. Lett. 39, 299 (1997); I.L. Aleiner et al., Phys. Rev. Lett. 79, 3740 (1997); S.A. Gurvitz, Phys. Rev. B 56, 15215 (1997).

8. E. Buks et al., Nature, Vol. 391, 871 (1998).

9. A.J. Leggett et al., Rev. Mod. Phys. 59, 1 (1987); U. Weiss, Quantum dissipative systems, World Scientific, Singapore, 1993.

10. H. Schoeller and G. Schön, Phys. Rev. B 50, 18436 (1994).

11. V. Bouchiat, D. Vion, P. Joyez, D. Esteve, and M.H. Devoret, to appear in Physica Scripta T176 (1998).

Acknowledgments. We thank E. Ben-Jacob, C. Bruder, E. Buks, L. Dreher, Z. Hermon, J. König, Y. Levinson, J.E. Mooij, T. Pohjola, and H. Schoeller for stimulating discussions. 